\title{
Shift work and 20-year incidence of acute myocardial infarction: results from the Kuopio Ischemic Heart Disease Risk Factor Study
}

\author{
Aolin Wang, ${ }^{1}$ Onyebuchi A Arah, ${ }^{1,2,3}$ Jussi Kauhanen, ${ }^{4}$ Niklas Krause ${ }^{1,5}$
}

- Additional material is

published online only. To view please visit the journal online (http://dx.doi.org/10.1136/ oemed-2015-103245).

${ }^{1}$ Department of Epidemiology, Fielding School of Public Health, University of California, Los Angeles (UCLA), Los Angeles, California, USA

${ }^{2}$ Center for Health Policy Research, UCLA, Los Angeles, California, USA

${ }^{3}$ California Center for Population Research, UCLA Los Angeles, California, USA ${ }^{4}$ Institute of Public Health and Clinical Nutrition, University of Eastern Finland, Kuopio,

Finland

${ }^{5}$ Department of Environmental Health Sciences, The Fielding School of Public Health, UCLA, Los Angeles, California, USA

\section{Correspondence to}

Dr Niklas Krause, Department of Environmental Health

Sciences and Department of Epidemiology, Fielding School of Public Health, UCLA, Box 95-1772; 56-071 CHS, 650 Charles E. Young Drive South, Los Angeles, CA 90095-1772, USA; niklaskrause@ucla.edu

Received 7 August 2015 Revised 1 March 2016 Accepted 10 March 2016

\footnotetext{
To cite: Wang $A$, Arah $O A$, Kauhanen J, et al. Occup Environ Med Published Online First: [please include Day Month Year] doi:10.1136/oemed-2015103245
}

\begin{abstract}
Objectives It remains unclear whether different types of shift work impose similar risks for cardiovascular events in middle-aged workers, especially those with pre-existing ischaemic heart disease (IHD). This study investigated the relations between different shift types and incident acute myocardial infarction (AMI) among men with and without pre-existing IHD, respectively.

Methods We analysed data on 1891 men, aged 4260 years at baseline, in the prospective Kuopio Ischemic Heart Disease Risk Factor Study cohort, using Cox proportional hazard models with adjustment for demographic, biological, behavioural and psychosocial job factors. We evaluated the associations of baseline shift work with 20-year incidence of AMI, and their modification by pre-existing IHD, using both stratified analysis and models with product terms between shift work and IHD.
\end{abstract}

Results Travelling work (at least 3 nights per week away from home) was strongly positively associated with AMl among men with $\mathrm{HD}(\mathrm{HR}=2.45,95 \% \mathrm{Cl} 1.08$ to 5.59) but not among men without $(\mathrm{HR}=0.93,95 \% \mathrm{Cl}$ 0.43 to 2.00). No clear associations were found between other types of shift work and AMI for both men with and without IHD. On both additive and multiplicative scales, baseline IHD status positively modified the association of travelling work with AMI (relative excess risk for interaction $=3.23,95 \% \mathrm{Cl}-0.50$ to $6.97, \mathrm{p}$ for multiplicative interaction $=0.044$ ).

Conclusions We found mixed results for the associations between different types of shift work and AMI among those with and without pre-existing IHD. Future research should investigate these associations and effect modification for a broad spectrum of work schedules.

\section{INTRODUCTION}

In this $24 \mathrm{~h}$ world, atypical working hours are increasingly prevalent. In Europe, more than half of the employees work at least 1 day during weekends, and about one in five workers perform night work, with men having a higher prevalence of night work. ${ }^{1}$ Shift work has been shown to increase risks for cardiovascular events. ${ }^{2}$ Several interrelated pathways have been proposed, including psychosocial stress, behavioural stress and physiological stress and reactions. ${ }^{3}$ Shift work may cause sleep deprivation and problems in family life (and subsequently inducing stress), change workers' behaviour

\section{What this paper adds}

- Mixed results have been documented for the association between shift work and cardiovascular events.

- Except for evening, night and rotating shifts, evidence is scant for increasingly common work schedules such as 'weekend shift' and 'travelling work'.

- This study found a near threefold increase in risk for acute myocardial infarction associated with travelling work among men with pre-existing ischaemic heart disease (IHD), but inconclusive results with other types of schedules depending on IHD status.

- Further research needs to address a broad spectrum of work schedules and investigate the possible modification of their health effects by workers' baseline cardiovascular health.

such as starting to smoke and developing unhealthy eating habits, leading to weight gain or change blood cholesterol levels, ${ }^{3}{ }^{4}$ all of which can increase the risk of cardiovascular disease (CVD).

Previous studies focused mainly on evening shifts, night shifts and rotating shifts, though the definition of shift work varied across studies. ${ }^{2}$ Evidence is scant for the full spectrum of work schedules. Workers may leave shift work within a few years due to management changes, changes in the social value assigned to shift work or health problems. ${ }^{5}$ These workers may not always end up doing standard daytime work. It is possible that workers leave shift work that involves evening and night for irregular or weekend shifts. It is still unclear within the broad umbrella of work schedules whether shifts that do not involve work during evening or night impose a similar risk for cardiovascular disease as evening/night/rotating shifts do. Also, only a few studies examined the impact of shift work on older workers. ${ }^{6-9}$ As workers age, they may be more vulnerable to the negative impact of shift work such as having more sleep problems with night shifts compared to younger workers. ${ }^{10}$

In this ageing working population in Eastern Finland, pre-existing ischaemic heart disease is highly prevalent among middle-aged workers. On the one hand, workers who have pre-existing ischaemic heart disease (IHD) are at higher risk for 
future coronary events and they may have already changed their behaviours. On the other hand, as predicted by the pathophysiological haemodynamic theory ${ }^{11}$ and also suggested in our previous papers, men with pre-existing IHD bear an excess risk for progression of atherosclerosis associated with shift work, ${ }^{12}$ standing work posture ${ }^{13}$ and high levels of occupational physical activity, ${ }^{14}$ and for incident AMI associated with occupational physical activity. ${ }^{15}$ It is important to know whether workers with pre-existing IHD experience higher risk for future AMI associated with certain types of shift work and thus should be counselled to work alternative schedules.

Using standard daytime workers as the reference group, this study investigates whether different types of shift work are associated with first-time incident acute myocardial infarction (AMI) during follow-up among men with IHD and those without, adjusting for an extensive list of baseline covariates that are known to be risk factors for AMI and may differ between day and shift workers. We examined several different types of schedules including a new category 'weekend work' that we introduced in a previous paper ${ }^{12}$ and 'travelling work' (at least 3 nights per week away from home) that has not been studied before. We hypothesised that men with pre-existing IHD would have a higher risk for future AMI events associated with shift work.

\section{METHODS}

\section{Study design, setting and population}

Participants were from the prospective Kuopio IHD Risk Factor (KIHD) Study, an age-stratified, random, population-based sample of Eastern Finnish men, residing in the city of Kuopio or its surrounding rural communities. Women were also recruited in the KIHD study recently but were not included in current analyses due to the short follow-up period. Details of the study population can be found elsewhere. ${ }^{15} 16$ Of the 3433 eligible men aged $42,48,54$ or 60 years, 198 were excluded due to death, serious illness or migration away from the area. Of the remaining men, $2682(82.9 \%)$ agreed to participate and underwent baseline examinations and interviews between March 1984 and December 1989. In this study, we excluded 791 participants who were not working during the 12 months prior to or at baseline, resulting in a final study sample of 1891 participants with complete information on all the covariates. All participants provided written informed consent. This study has been approved by the University of California, Los Angeles Institutional Review Board.

\section{Assessment of incidence of AMI}

As described previously, ${ }^{15}$ we ascertained first-time incident AMI (International Classification of Diseases Ninth Edition, ICD-9 code 410) during follow-up via record linkage with national hospitalisation discharge and death registries including the national AMI register established under the WHO's "Monitoring of Trends and Determinants of Cardiovascular Diseases (MONICA)" project. ${ }^{17}{ }^{18}$ A university-based cardiologist for this study confirmed hospital discharge diagnoses using other hospital records, lab results and ECGs. We censored the follow-up at December 31, 2011 or date of death, whichever came first.

\section{Assessment of work schedules}

Participants reported work schedules and the number of days worked per week in the self-administered questionnaire at baseline. Given the previously found positive association between work time and progression of atherosclerosis ${ }^{19}$ and the positive association between weekend work and progression of atherosclerosis, ${ }^{12}$ we separated people who did standard daytime work (worked during weekdays) from those who also worked on weekends (ie, $>5$ days/week). We created two types of work schedule definitions: a binary definition and a multinomial definition. The binary definition classifies people as doing any shift work if they reported working non-standard daytime hours (including working on weekends). The reference group was standard daytime work-people who reported working during daytime only and working $\leq 5$ days per week. The multinomial definition further categorised any shift work as follows: (1) weekend shifts -people who worked during daytime and more than 5 days per week; (2) evening/night/rotating shifts-including people doing evening work, night work or rotating shift (work schedules that alternate between day, evening and night); (3) two-shift workwork schedules that alternate between day and evening; (4) irregular work (also called 'swing shift' in Finland in the late 1980s); (5) travelling work (at least 3 nights per week away from home, also called 'work on demand'). Categories (2) to (5) were created regardless of the number of days worked per week.

\section{Assessment of covariates}

We included the following groups of covariates measured at baseline, the details of which have been described previously. ${ }^{20} 21$

Age and technical factors: age and participation in an unrelated lipid-lowering drug trial.

Socioeconomic status was measured using annual personal income (in 1000 FIM).

Biological factors: blood samples were drawn to determine blood glucose and plasma fibrinogen levels, serum low-density lipoprotein cholesterol, serum high-density lipoprotein cholesterol (HDL). Body mass index was derived from measured height and weight (weight/height ${ }^{2}, \mathrm{~kg} / \mathrm{m}^{2}$ ). Systolic blood pressure and whether the patient took lipid-lowering or blood pressure-lowering medication during follow-up were also included. Cardiorespiratory fitness $\left(\mathrm{VO}_{2} \max \right)$ was measured with a maximal, symptom-limited exercise-tolerance test on a bicycle ergometer.

Behavioural factors: Smoking, alcohol and leisure-time physical activity were assessed by a questionnaire. A continuous smoking variable 'pack-years' was calculated as the number of packs (20 cigarettes/pack) per day times the number of years smoked. Alcohol consumption (grams per week) accounted for frequency of drinking and amount of drinks per occasion for each type of alcoholic beverage (beer, wine and spirits) for the past 12 months. Conditioning (moderate or vigorous) leisuretime physical activity (hours per year) was measured using a modified version of the Minnesota Leisure Time Physical Activity questionnaire. ${ }^{22}$

Psychosocial job factors were measured by a questionnaire including social support at work (3 items), mental strain at work (11 items of psychological demands) and stress from work deadlines and showed satisfactory Cronbach's $\alpha$ coefficients. ${ }^{21} 23$

\section{Assessment of baseline IHD}

We classified participants as having pre-existing IHD at baseline if, based on self-report, they (1) had a history of prior (before baseline) myocardial infarction or angina pectoris, (2) currently used antiangina medication or (3) had positive findings of angina according to the London School of Hygiene cardiovascular questionnaire. ${ }^{24}$

\section{Statistical analysis}

We summarised the participants' characteristics by their baseline IHD status. Then we used the Cox proportional hazard (PH) models $^{25}$ with incremental adjustment of covariates listed in 
table 1. Separately for men without and with IHD, the PH assumption was tested using the goodness of fit testing approach based on Schoenfeld residuals and was not rejected by this global test when including all variables listed in table 1 in the Cox model (stphtest command in Stata). ${ }^{26}$ We also performed the $\mathrm{PH}$ test for each covariate separately (detailed option) and the null hypothesis of $\mathrm{PH}$ was rejected among men without baseline IHD for the following covariates: participation in an unrelated lipid-lowering drug trial, HDL cholesterol and smoking, using 0.05 as the cut-off point. We used a stratified Cox (SC) model for men without IHD and obtained similar results to those from the regular Cox model. We present results from the regular Cox model for both men with and without baseline IHD in the main text for consistency and included results from the SC model in an online supplementary file.

The smoking measure captured the cumulative smoking behaviour over the lifetime. Measures for alcohol consumption and leisure-time physical activity represented behaviours over the past 12 months. Psychosocial job factors, though measured at baseline, may correlate closely with job stress factors before baseline. Thus, adjusting for these factors could account for differences in participant characteristics that may be due to factors other than work schedule, as is often done in the literature. Biological factors may be considered as potential mediators on the pathway from shift work to AMI. ${ }^{4}$ Nevertheless, adjustment for such factors may still be necessary if they are transmitting the impact of an unmeasured confounder such as job function or chronotypes ('morning people' vs 'night owls') on future AMI. Further adjustment will control for confounding due to this unmeasured factor. The estimates will be the controlled direct effect of work schedule on AMI (ie, holding the level of these mediating factors at their mean or reference values) instead of the total effect, given that there is no uncontrolled confounding between these biological, behavioural and psychosocial job factors and AMI conditional on all the factors we account for. The adjustment for baseline biological, behavioural and psychosocial job factors, however, will not exclude any influence of shift work on AMI that is exerted via changes in

Table 1 Characteristics of the study sample and distribution of exposure and covariates by IHD status at baseline, Kuopio Ischemic Heart Disease Risk Factor Study, 1984-2011 (N=1891)

\begin{tabular}{|c|c|c|c|c|c|c|c|c|}
\hline \multirow[b]{2}{*}{ Variable } & \multicolumn{4}{|c|}{ Men without IHD ( $\mathrm{N}=1565)$} & \multicolumn{4}{|c|}{ Men with IHD $(\mathrm{N}=326)$} \\
\hline & Mean & SD & $\mathrm{N}$ & Per cent & Mean & SD & $\mathrm{N}$ & Per cent \\
\hline \multicolumn{9}{|l|}{ Work schedules } \\
\hline Standard daytime work & & & 1027 & 65.6 & & & 198 & 60.7 \\
\hline Weekend shifts & & & 276 & 17.6 & & & 76 & 23.3 \\
\hline Evening/night/rotating shifts & & & 60 & 3.8 & & & 12 & 3.7 \\
\hline Two shifts & & & 74 & 4.7 & & & 9 & 2.8 \\
\hline Irregular work & & & 95 & 6.1 & & & 20 & 6.1 \\
\hline Travelling work & & & 33 & 2.1 & & & 11 & 3.4 \\
\hline \multicolumn{9}{|l|}{ Covariates } \\
\hline \multicolumn{9}{|l|}{ Age and technical factors } \\
\hline Age at baseline (years) & 51.5 & 5.1 & & & 53.5 & 3.9 & & \\
\hline \multicolumn{9}{|l|}{ Participation in a lipid-lowering drug trial } \\
\hline Placebo group & & & 135 & 8.6 & & & 28 & 8.6 \\
\hline Treatment group & & & 136 & 8.7 & & & 27 & 8.3 \\
\hline \multicolumn{9}{|l|}{ Socioeconomic status } \\
\hline Annual personal income (1000 FIM) & 92.6 & 56.7 & & & 72.3 & 37.4 & & \\
\hline \multicolumn{9}{|l|}{ Biological factors } \\
\hline Blood glucose level (mmol/L) & 4.7 & 0.9 & & & 4.9 & 1.4 & & \\
\hline Plasma fibrinogen level (g/L) & 2.9 & 0.5 & & & 3.1 & 0.6 & & \\
\hline BMI at baseline $\left(\mathrm{kg} / \mathrm{m}^{2}\right)$ & 26.7 & 3.4 & & & 27.1 & 3.8 & & \\
\hline LDL cholesterol at baseline (mmol/L) & 4.0 & 1.0 & & & 4.2 & 1.1 & & \\
\hline HDL cholesterol at baseline (mmol/L) & 1.3 & 0.3 & & & 1.3 & 0.3 & & \\
\hline SBP at baseline $(\mathrm{mm} \mathrm{Hg})$ & 133.9 & 16.0 & & & 133.0 & 18.3 & & \\
\hline Taking lipid-lowering medication & & & 4 & 0.3 & & & 4 & 1.2 \\
\hline Taking blood pressure-lowering medication & & & 179 & 11.4 & & & 115 & 35.3 \\
\hline Cardiorespiratory fitness $\left(\mathrm{ml} \mathrm{O}_{2} / \mathrm{kg} / \mathrm{min}\right)$ & 32.8 & 7.0 & & & 27.5 & 6.9 & & \\
\hline \multicolumn{9}{|l|}{ Behavioural factors } \\
\hline Smoking (pack-years) & 7.1 & 14.5 & & & 10.5 & 17.4 & & \\
\hline Alcohol consumption (g/week) & 71.5 & 111.8 & & & 88.3 & 196.4 & & \\
\hline Conditioning LTPA (hours/year) & 90.9 & 101.8 & & & 94.6 & 125.6 & & \\
\hline \multicolumn{9}{|l|}{ Psychosocial job factors } \\
\hline Social support at work score* & 11.5 & 6.3 & & & 13.4 & 7.1 & & \\
\hline Mental strain at work index $\dagger$ & 6.5 & 2.5 & & & 6.5 & 2.4 & & \\
\hline Stress from work deadlines & & & 357 & 22.8 & & & 105 & 32.2 \\
\hline
\end{tabular}

*Higher score means less social support.

tHigher score means experiencing more mental strain.

BMI, body mass index; FIM, Finnish markka; HDL, high-density lipoprotein; IHD, ischaemic heart disease; LDL, low-density lipoprotein; LTPA, leisure-time physical activity; SBP, systolic blood pressure. 
these factors during follow-up. Therefore, in the main manuscript, we presented a crude model, a model adjusting only for age and technical factors, and a fully adjusted model. In the supplementary file, we presented intermediate models with adjustment for biological, behavioural and psychosocial job factors, respectively, on top of the age-adjusted and technical factors-adjusted model.

We also used a model with product terms for shift work by IHD status for all men combined to assess statistically whether the shift work-AMI associations differed by IHD status, that is, examining potential effect measure modification or statistical interaction by pre-existing IHD. A significant non-zero product term would indicate IHD modifies the shift work-AMI association on the multiplicative scale. We also calculated the relative excess risk for interaction (RERI) as a measure for additive interaction, which indicates biological interaction. ${ }^{27}$ RERI $>0$ would indicate that the combined effect of shift work and IHD is greater than the sum of their separate effects assuming monotonic effects of both exposures. ${ }^{28}$ HRs and their corresponding 95\% CI were reported separately for men with and without IHD. All analyses were performed using Stata V.14 (StataCorp LP, College Station, Texas, USA).

\section{Sensitivity analyses}

Since some of the oldest workers in the current cohort may have stopped working a few years after baseline, we repeated our main analysis by (1) censoring participants on the date 5 years after their retirement date or June 1, 2000 (when the retirement information was obtained) and (2) excluding workers aged 60 years or older at baseline. In addition to the covariates listed in table 1 , we conducted separate analyses with further adjustment for the following covariates: (1) hours worked per week (excluding breaks) at baseline, (2) occupational physical activity (measured as relative aerobic $\operatorname{strain}^{14}$ ) and (3) preexisting diabetes.

\section{RESULTS}

During an average of 19.56 years of follow-up (SD 7.53, range 0.01-27.76) and a total person time of 36991 years, 495 firsttime incident AMI occurred among 1891 study participants, yielding a yearly incidence rate of $1.34 \%$. Among 1565 men without baseline IHD, 353 AMI occurred (yearly incidence 1.11\%), whereas among 326 men with baseline IHD, 142 AMI occurred (yearly incidence $2.60 \%$ ).

In our sample, $35 \%$ of men reported doing any type of shift work, including work during weekend $(n=352)$, evening $(n=5)$, night $(n=8)$, rotating shifts $(n=59)$, two shifts $(n=83)$, irregular shifts $(n=115)$ or travelling work $(n=44)$. Compared to men without baseline IHD, men with IHD were more likely to work during non-standard daytime hours, especially during weekends and away from home (travelling work). They were also slightly older, more likely to take lipid-lowering or blood pressurelowering medication, had lower levels of cardiorespiratory fitness, drank and smoked more and earned less money (table 1). The distribution of work schedules by main occupation is presented in the online supplementary table S1. Briefly, weekend shifts were concentrated in the agricultural, forestry and commercial fishing industries (69\%) followed by sales (9\%), evening/ night and rotating shifts in the manufacturing sector (39\%) followed by technical/science/human service work (24\%); two shifts were most common in manufacturing (43\%) followed by transportation and communication (29\%); irregular work was most frequent in transportation and communication (39\%) and agriculture/forestry/fishing (17\%), and travelling work was common in sales $(39 \%)$ followed by manufacturing/construction/manual labor (27\%).

Table 2 depicts the associations between shift work and AMI incidence, stratified by IHD status. No associations were found between the binary shift work indicator and AMI, regardless of IHD status. Among men without IHD, a positive yet nonsignificant association was found between two shifts and AMI $(\mathrm{HR}=1.47,95 \%$ CI 0.93 to 2.31 ), whereas no associations were found between other types of shift work and AMI. Results from the stratified Cox model for men without IHD showed similar results (see online supplementary table S2). We observed a strong positive association of travelling work with AMI among men with pre-existing IHD ( $\mathrm{HR}=2.45$, 95\% CI 1.08 to 5.59$)$, but other types of shift work were not associated with AMI in this subgroup. Results from the intermediate models were presented in online supplementary table S3. Estimates fluctuated slightly, indicating possible cancellation of confounding due to different factors.

Table 3 depicts the model-based IHD status-specific fully adjusted estimates for shift work-AMI associations and the corresponding RERI and $\mathrm{p}$ value for multiplicative interaction. Positive additive and multiplicative interaction was found between travelling work and baseline IHD status (RERI=3.23, $95 \%$ CI -0.50 to $6.97, \mathrm{p}$ for multiplicative interaction $=0.044$ ). Yet no statistical interactions were found between IHD and work schedules other than travelling work (RERI ranges from -0.53 to 0.14 , $\mathrm{p}$ for multiplicative interaction $>0.20)$. The model-based estimates were similar to those from the stratified analyses presented in table 2 .

Results from sensitivity analyses were presented in online supplementary table S4-S5. Similar results to those from table 2 were found after censoring participants based on their retirement date, excluding men aged 60 years or older, or additionally adjusting for work hours, occupational physical activity, or pre-existing diabetes.

\section{DISCUSSION}

This study examined the associations between different work schedules and AMI incidence during a 20-year follow-up of men with and without pre-existing IHD. Over two times elevated risk for AMI was linked to travelling work among men with pre-existing IHD but not among those without. Two-shift work was weakly positively associated with AMI among men without IHD, though the estimate was uncertain. Other work schedules such as weekend shifts, evening/night/rotating shifts or irregular work showed only weak and uncertain associations with AMI after accounting for baseline covariates.

According to a recent meta-analysis, all types of shift work were associated with a $23 \%$ increase in risk of myocardial infarction (MI) overall based on 10 studies. ${ }^{2}$ Among these studies, four cohort studies (both prospective and retrospective) found elevated risk in $\mathrm{MI}$ associated with rotating shift, ${ }^{29}$ irregular shift ${ }^{30}$ and mixed shift (defined as 'ever worked in shift and night work' in one study ${ }^{31}$ and 'having shift work, night work or stand-by duties' in the other ${ }^{32}$ ). Effect modification by pre-existing IHD status is of particular concern among older workers as workers with IHD have a higher risk for additional coronary events. The different definitions of shift work make it difficult to compare our results directly to the above studies. In our sample, neither all shift work schedules combined nor the subcategories of evening/night/rotating shifts or irregular shifts seem to be associated with AMI in the fully adjusted model. However, a more than doubled risk of AMI was observed for travelling workers with IHD. We also observed a 
Table 2 HRs and $95 \% \mathrm{Cls}$ of the associations between work schedule and 20-year incidence of acute myocardial infarction ( $\mathrm{N}=495)$ for men without and men with IHD, Kuopio Ischemic Heart Disease Risk Factor Study, 1984-2011 ( $N=1891)$

\begin{tabular}{|c|c|c|c|c|c|c|}
\hline \multirow[b]{2}{*}{ Work schedule } & \multicolumn{3}{|c|}{ Men without IHD ( $\mathrm{N}=1565)$} & \multicolumn{3}{|c|}{ Men with IHD (N=326) } \\
\hline & Crude & $\begin{array}{l}\text { Age and technical } \\
\text { factors adjusted }\end{array}$ & Fully adjusted* & Crude & $\begin{array}{l}\text { Age and technical } \\
\text { factors adjusted }\end{array}$ & Fully adjusted* \\
\hline \multicolumn{7}{|l|}{ Binary definition } \\
\hline Standard day work & 1 & 1 & 1 & 1 & 1 & 1 \\
\hline Any shift work & 1.09 (0.88 to 1.36$)$ & $1.08(0.87$ to 1.35$)$ & $1.09(0.87$ to 1.37$)$ & $0.96(0.69$ to 1.35$)$ & 0.96 (0.68 to 1.34$)$ & 1.05 (0.73 to 1.50$)$ \\
\hline \multicolumn{7}{|l|}{ Multinomial definition } \\
\hline Standard day work & 1 & 1 & 1 & 1 & 1 & 1 \\
\hline Weekend shifts & 1.19 (0.91 to 1.57$)$ & $1.14(0.87$ to 1.50$)$ & $1.13(0.84$ to 1.50$)$ & $1.06(0.72$ to 1.57$)$ & 1.02 (0.68 to 1.52$)$ & 1.08 (0.71 to 1.65$)$ \\
\hline Evening/night/rotating shifts & $0.76(0.40$ to 1.43$)$ & $0.82(0.43$ to 1.54$)$ & $1.01(0.53$ to 1.91$)$ & $0.70(0.26$ to 1.91$)$ & 0.75 (0.27 to 2.07$)$ & 0.83 (0.25 to 2.74$)$ \\
\hline Two shifts & 1.35 (0.87 to 2.12$)$ & 1.45 (0.93 to 2.28$)$ & $1.47(0.93$ to 2.31$)$ & 0.75 (0.24 to 2.36$)$ & $0.82(0.26$ to 2.60$)$ & 0.93 (0.29 to 3.04$)$ \\
\hline Irregular work & $0.89(0.56$ to 1.40$)$ & 0.86 (0.55 to 1.36$)$ & 0.90 (0.57 to 1.44$)$ & 0.52 (0.21 to 1.28$)$ & 0.52 (0.21 to 1.28$)$ & 0.60 (0.23 to 1.52$)$ \\
\hline Travelling work & $1.06(0.50$ to 2.26$)$ & 1.06 (0.50 to 2.24$)$ & $0.93(0.43$ to 2.00$)$ & 1.94 (0.90 to 4.19$)$ & $2.11(0.94$ to 4.71$)$ & 2.45 (1.08 to 5.59$)$ \\
\hline
\end{tabular}

${ }^{*}$ Model adjusted for all covariates listed in table 1.

IHD, ischaemic heart disease.

higher risk estimate for AMI associated with two shifts compared to evening/night/rotating shifts among men without IHD. This is inconsistent with a previous study that found that industrial workers in two-shift and three-shift work have similar elevated risk for coronary heart disease (CHD), ${ }^{8}$ and our finding awaits an explanation and confirmation in future studies.

Cohort studies that focus on middle-aged working populations are limited. One cohort study examined the impact of shift work on CHD in a middle-aged (40-55 years at entry) Finnish male industrial population ${ }^{8}$ and found a risk ratio of 1.33 (95\% CI 0.94 to 2.08) comparing permanent shift workers to permanent day workers, and this relation did not change much (RR 1.59 , 95\% CI 0.70 to 3.61 ) after excluding participants receiving cholesterol-lowering medication or having been previously diagnosed with cardiovascular disease. A study from the Finnish Twin Cohort ${ }^{9}$ with a mean age of 40 years had a similar study population, study period and covariate adjustment scheme as ours and found no increase in risk of mortality due to $\mathrm{CHD}$, disability retirement due to CVD or incident hypertension comparing shift workers to daytime workers. However, this study found mixed results in the associations between night-time work in 1975 or 1981 and the aforementioned three outcomes.
Another study on mortality among male manual workers ${ }^{7}$ also found no excess mortality in either the shift or ex-shift groups compared to day workers. In addition, shift work, including working irregular hours and night work, was found to be not independently predicting IHD when accounting for social class. $^{33}$ These findings of null associations among middle-aged men may be mainly due to collapse of different shift categories, possible outcome misclassification or underadjustment or overadjustment (lack of control for important confounders or adjusting for potential mediators, respectively). It is also possible that the impact of shift work differs in the younger population versus older population, with the latter being a potential survivor group.

The pattern we observed for different shift categories by IHD status may be partially explained by differential selection into and out of certain shift categories. For example, evening and night shift workers with IHD may change their work schedules if they were more aware about their potential risk of future AMI than their counterparts who did other types of shifts or day work. Their elevated risk for AMI associated with evening or night work (ie, not accounted for by covariate adjustment) will then be imported into the new work schedule they choose.

Table 3 Fully adjusted IHD status-specific HRs* and 95\% Cls of the associations between work schedule and 20-year incidence of acute myocardial infarction ( $\mathrm{N}=495)$ for all men, Kuopio Ischemic Heart Disease Risk Factor Study, 1984-2011 ( $\mathrm{N}=1891)$

\begin{tabular}{|c|c|c|c|c|}
\hline Work schedule & $\begin{array}{l}\text { No IHD } \\
\text { HR }(95 \% \mathrm{Cl})\end{array}$ & $\begin{array}{l}\text { With IHD } \\
\text { HR }(95 \% \mathrm{Cl})\end{array}$ & RERI $(95 \% \mathrm{Cl}) \dagger$ & $\begin{array}{l}p \text { Value for multiplicative } \\
\text { interaction } \neq\end{array}$ \\
\hline \multicolumn{5}{|l|}{ Binary definition } \\
\hline Standard day work & 1 & 1 & 0 & - \\
\hline Any shift work & 1.09 (0.87 to 1.37$)$ & 0.97 (0.69 to 1.37 ) & $-0.14(-0.74$ to 0.46$)$ & 0.573 \\
\hline \multicolumn{5}{|l|}{ Multinomial definition } \\
\hline Standard day work & 1 & 1 & 0 & - \\
\hline Weekend shifts & 1.14 (0.86 to 1.51$)$ & $0.90(0.60$ to 1.35$)$ & $-0.30(-0.98$ to 0.38$)$ & 0.344 \\
\hline Evening/night/rotating shifts & 0.91 (0.48 to 1.72$)$ & $1.03(0.37$ to 2.90$)$ & $0.14(-1.66$ to 1.94$)$ & 0.840 \\
\hline Two shifts & 1.45 (0.92 to 2.28$)$ & $1.19(0.37$ to 3.79$)$ & $-0.15(-2.45$ to 2.14$)$ & 0.751 \\
\hline Irregular work & $0.91(0.58$ to 1.45$)$ & $0.62(0.25$ to 1.53$)$ & $-0.53(-1.58$ to 0.52$)$ & 0.448 \\
\hline Travelling work & 0.98 (0.46 to 2.08$)$ & 2.99 (1.36 to 6.57$)$ & $3.23(-0.50$ to 6.97$)$ & 0.044 \\
\hline
\end{tabular}

*Based on models that included work schedule, IHD status, and work schedulexIHD product terms. Model adjusted for all covariates listed in table 1. TRERIs were measures for additive interaction and were calculated on the basis of coefficients from fully adjusted models with work schedulexIHD product terms. $\neq p$ Value for each work schedulexIHD product term.

IHD, ischaemic heart disease; RERI, relative excess risk for interaction. 
Although the time at which such changes would have happened is not clear, it is less likely to happen during our follow-up period because job stability was quite high among middle-aged men in Finland when this study was carried out. ${ }^{34}$ Also, on the basis of information of beginning and ending time at work, the typical daily working time period appeared to be stable in this population. In a subgroup of 553 men from our study, $96 \%$ of these men began their work at 4-year follow-up at a time within $\pm 2 \mathrm{~h}$ of the time reported at baseline and $89 \%$ of these men ended their work at 4-year follow-up at a time within $\pm 2 \mathrm{~h}$ of the time reported at baseline, indicating a relatively stable work schedule among these men. Further, men who had IHD at an early age may differ in other aspects with regard to the risk for future AMI such as risks associated with extended work time ${ }^{19}$ and occupational physical activity. ${ }^{14}{ }^{15}$ However, the pattern remained the same even after further adjusting for these two risk factors measured at baseline (see online supplementary table S5).

The increased AMI risk for travelling workers with IHD found in this study is interesting. We also found that the combined effect of travelling work and pre-existing IHD on future AMI is greater than the sum of their separate effects. This finding supports our hypothesis that ageing workers with prevalent IHD at baseline would be more vulnerable to the detrimental impact of travelling work in excess of their higher risk for a an additional cardiac event. Travelling work was defined as staying away from home for at least 3 days per week and may therefore have similar negative impacts in terms of social disruption like weekend or night shifts. ${ }^{35-37}$ Also, travelling work more likely entails longer hours of commuting, extended work hours including travelling, more stress and chronic fatigue. In our sample, travelling workers had a much longer distance between home and job (mean round trip distance $=55 \mathrm{~km}$ ) compared to other shift categories (mean round trip distance ranging from $6 \mathrm{~km}$ for weekend workers to $17 \mathrm{~km}$ for workers doing day, evening/night/rotating shift or irregular shift). Accordingly, $82 \%$ of these travelling workers went to work by car or taxi. Regular car commuting was linked to increased risk of myocardial infarction versus commuting by bus, cycling or walking $^{38}$ and to perceived poor sleep quality, low self-rated health and low vitality compared to active commuting (cycling or walking only). ${ }^{39}$ Managing long car commuting could be more challenging for aged workers, as older workers ( $\geq 45$ years) are found to be in greater need for recovery after work compared to younger workers $\left(<45\right.$ years) ${ }^{40}$ perhaps even more so among workers with existing IHD, though a decreased need for recovery was seen among employees aged 55 years or older in another study. ${ }^{41}$ Long working hours ${ }^{42}$ and heavy occupational physical activity ${ }^{15}$ have been shown to increase the risk of cardiovascular events. According to our sensitivity analyses, the elevated risk for travelling workers with IHD is robust against additional covariate adjustment for these two factors. Further adjusting for pre-existing diabetes did not change the positive association between AMI and travelling work among workers with IHD. Among the 14 workers with both pre-existing IHD and diabetes, 13 worked the standard daytime or weekend shift. This suggests that middle-aged workers who had pre-existing chronic diseases such as diabetes may have already selected themselves out of shift work before the study period. Owing to the relatively small number of participants in this category, we call for further larger studies, especially among those with pre-existing conditions.

In our previous study on shift work and ultrasonically measured 11-year progression of carotid atherosclerosis, we found weekend shift workers to experience a faster progression of atherosclerosis and an even faster progression among men with pre-existing IHD. ${ }^{12}$ In contrast, in the current study, weekend shifts were not associated with AMI among men with IHD and associated with an uncertain 14\% increased risk among men without IHD. This inconsistent pattern of findings may be due to health-based selection effects among men with IHD: in contrast to asymptomatic carotid atherosclerosis, angina pectoris symptoms may reduce tolerance of stressful working conditions and any awareness of potential increased disease risks associated with shift work (including the weekend shift) may have prompted those workers or their treating physicians to seek job or work schedule change before any AMI occurs, thus diluting the apparent risk of AMI for weekend shift.

Our study has several strengths. First, we studied a representative general population sample rather than a cohort of shift workers. Thus, our results cannot be accounted for by other occupational risks that may be related to a single line of business, and our findings may apply to middle-aged men in general. Second, we used incident AMI during follow-up ascertained by hospital records as the outcome, reducing potential outcome misclassification due to self-report. Third, we adjusted for an extensive list of baseline covariates, allowing proper comparisons between standard daytime work and different shift schedules. Fourth, selection bias due to loss of follow-up was unlikely, as outcome status was ascertained for every cohort member. Fifth, analyses of detailed work schedules allowed for discovering levels of AMI risk associated with different work schedules. In fact, our study demonstrated that combining different shift schedules into one binary exposure variable ('any shift work') can mask even strong risks associated with specific work schedules like the 2.5 -fold AMI risk for travelling work. Finally, stratification by baseline IHD helped identify high-risk groups for shift work-related AMI that would have been missed in analyses of all men together (see online supplementary table S6). Failure to stratify analyses by potential effect modifiers such as baseline IHD status may lead to equivocal or inconsistent results that plague the literature on occupational risk factors and CVD in general, and shift work and CVD in particular.

On the other hand, stratification by IHD status led to relative small sample sizes in each subgroup and limited statistical power. Lack of work schedule history throughout working life prohibited us from examining the intensity of shiftwork. Additional details of shift schedules (number of night shifts in a row, rotating direction, etc) ${ }^{43}$ were not available for further classification of shift types. The middle-aged shift workers may constitute a survivor population who can manage these nonstandard work schedules, which could attenuate effect sizes and limit generalisability of results. 'Secondary selection', where workers self-select out of certain shift types due to health concerns or existing health conditions, is a common concern in the shift work literature ${ }^{4}$ and can lead to differential exposure misclassification. Even though job stability was high among these workers, such bias may have occurred. Our data contained only a one-time assessment of work schedules, precluding further examination of the potential impact of such bias. Multiple comparisons between different work schedules and with different covariate adjustment schemes were made and chance findings might have arisen.

In conclusion, examination of 1225 middle-aged daytime workers and 666 middle-aged shift workers found an increased risk of 20-year AMI incidence associated with travelling work among men with IHD, but inconclusive results with other types 
of schedules. We provided convincing new evidence that associations between shift work and AMI can differ by pre-existing IHD. Further, to the best of our knowledge, this is the first study that identified travelling work to be a potential strong risk factor for future AMI among men with IHD. Future research needs to confirm these findings and-with sufficient sample size and variation in work schedules-explore different work schedules in greater detail. Assessing effect modification by age and preexisting conditions such as IHD will help to identify high-risk subpopulations and aid the development of specific interventions, especially for the increasing proportion of older workers.

Acknowledgements The authors thank Kimmo Ronkainen for managing and updating the underlying KIHD databases. The authors also thank cardiologist Jari Laukkanen for providing expert validation of AMI diagnosis by checking national hospital discharge registry data with other available hospital records including ECG and laboratory data.

Contributors AW conceived this study, performed all analyses and wrote the manuscript. NK participated in study conception, provided guidance on analysis and interpretation of the result, and edited the manuscript. JK served as a liaison with KIHD study data management at the University of Eastern Finland, provided access to data for this study, and reviewed the final manuscript. OAA provided statistical advice for model building and the interpretation of results, and reviewed and edited the final manuscript draft.

Funding AW was supported by the Dissertation Year Fellowship from the University of California, Los Angeles and a doctoral scholarship from the Chinese Scholarship Council (CSC). CDC/NIOSH grant number R01-OH010832-01 supported the contributions of OAA and NK. In addition, this research was partially funded by the Finnish Academy of Science through the University of Eastern Finland and by the Southern California NIOSH Education and Research Center (Award Number 5T42OH008412-09 from the Centers for Disease Control and Prevention (CDC)). Its contents are solely the responsibility of the authors and do not necessarily represent the official view of CDC

Competing interests None declared.

Patient consent Obtained.

Provenance and peer review Commissioned; internally peer reviewed.

\section{REFERENCES}

1 Eurofound. Fifth European Working Conditions survey. Luxembourg, 2012. http:// www.eurofound.europa.eu/sites/default/files/ef_files/pubdocs/2011/82/en/1/ EF1182EN.pdf

2 Vyas MV, Garg AX, lansavichus AV, et al. Shift work and vascular events: systematic review and meta-analysis. BMJ 2012;345:e4800.

3 Puttonen S, Harma M, Hublin C. Shift work and cardiovascular disease-pathways from circadian stress to morbidity. Scand I Work Env Heal 2010;36:96-108.

4 Boggild H, Knutsson A. Shift work, risk factors and cardiovascular disease. Scand I Work Env Heal 1999;25:85-99.

5 Boggild $H$. Settling the question - the next review on shift work and heart disease in 2019. Scand I Work Env Heal 2009;35:157-61.

6 Viitasalo K, Puttonen S, Kuosma E, et al. Shift rotation and age-interactions with sleep-wakefulness and inflammation. Ergonomics 2015;58:65-74.

7 Taylor PJ, Pocock SJ. Mortality of shift and day workers 1956-68. Br J Ind Med 1972;29:201-7.

8 Tenkanen L, Sjöblom T, Kalimo R, et al. Shift work, occupation and coronary heart disease over 6 years of follow-up in the Helsinki Heart Study. Scand I Work Environ Health 1997;23:257-65.

9 Hublin C, Partinen M, Koskenvuo K, et al. Shift-work and cardiovascular disease: a population-based 22-year follow-up study. Eur J Epidemiol 2010;25:315-23.

10 Blok MM, de Looze MP. What is the evidence for less shift work tolerance in older workers? Ergonomics 2011;54:221-32.

11 Glagov S, Zarins C, Giddens DP, et al. Hemodynamics and atherosclerosis. Insights and perspectives gained from studies of human arteries. Arch Pathol Lab Med 1988:112:1018-31.

12 Wang A, Arah OA, Kauhanen J, et al. Work schedules and 11-year progression of carotid atherosclerosis in middle-aged Finnish men. Am J Ind Med 2015;58:1-13.

13 Krause N, Lynch JW, Kaplan GA, et al. Standing at work and progression of carotid atherosclerosis. Scand I Work Env Heal 2000;26:227-36.

14 Krause N, Brand RJ, Kaplan GA, et al. Occupational physical activity, energy expenditure and 11-year progression of carotid atherosclerosis. Scand I Work Env Heal 2007:33:405-24.
15 Krause N, Brand RJ, Arah OA, et al. Occupational physical activity and 20-year incidence of acute myocardial infarction: results from the Kuopio Ischemic Heart Disease Risk Factor Study. Scand I Work Environ Health 2015;41:124-39.

16 Salonen JT. Is there a continuing need for longitudinal epidemiologic research? The Kuopio Ischaemic Heart Disease Risk Factor Study. Ann Clin Res 1988;20: 46-50.

17 Tunstall-Pedoe H, Kuulasmaa K, Amouyel P, et al. Myocardial infarction and coronary deaths in the World Health Organization MONICA Project. Registration procedures, event rates, and case-fatality rates in 38 populations from 21 countries in four continents. Circulation 1994;90:583-612.

18 Tuomilehto J, Arstila M, Kaarsalo E, et al. Acute myocardial infarction (AMI) in Finland-baseline data from the FINMONICA AMI register in 1983-1985. Eur Heart J 1992:13:577-87.

19 Krause N, Brand RJ, Kauhanen J, et al. Work time and 11-year progression of carotid atherosclerosis in middle-aged Finnish men. Prev Chronic Dis 2009;6:A13, $1-20$.

20 Lynch JW, Kaplan GA, Cohen RD, et al. Do cardiovascular risk factors explain the relation between socioeconomic status, risk of all-cause mortality, cardiovascular mortality, and acute myocardial infarction? Am J Epidemio/ 1996;144:934-42.

21 Lynch J, Krause N, Kaplan G, et al. Workplace conditions, socioeconomic status, and the risk of mortality and acute myocardial infarction: the Kuopio Ischemic Heart Disease Risk Factor Study. Am J Public Health 1997;87:307-25.

22 Taylor HL, Jacobs DR, Schucker B, et al. A questionnaire for the assessment of leisure time physical activities. J Chronic Dis 1978;31:741-55.

23 Lynch J, Krause N, Kaplan G, et al. Workplace demands, economic reward, and progression of carotid atherosclerosis. Circulation 1997:96:302-7.

24 Rose GA. The diagnosis of ischaemic heart pain and intermittent claudication in field surveys. Bull. World Health Organ. 1962;27:645-58.

25 Cox DR, Oakes D. Analysis of survival data. New York: Chapman \& Hall//CRC, 1984.

26 Kleinbaum DG, Klein M. Survival analysis—A self-learning text. Third Edit. New York, NY: Springer, 2011. http://www.springer.com/us/book/9781441966452

27 Rothman KJ, Greenland S, Lash TL. Modern epidemiology. 3rd edn. Philadelphia: Wolters Kluwer Health/Lippincott Williams \& Wilkins, 2008. http://www.loc.gov/ catdir/enhancements/fy0743/2007036316-d.html

28 Richardson DB, Kaufman JS. Estimation of the relative excess risk due to interaction and associated confidence bounds. Am J Epidemio/ 2009;169:756-60.

29 Kawachi I, Colditz GA, Stampfer MJ, et al. Prospective study of shift work and risk of coronary heart disease in women. Circulation 1995;92:3178-82. http://www. ncbi.nlm.nih.gov/entrez/query.fcgi?cmd=Retrieve\&db=PubMed\&dopt=Citation\&list_ uids $=7586301$

30 Alfredsson L, Spetz CL, Theorell T. Type of occupation and near-future hospitalization for myocardial infarction and some other diagnoses. Int I Epidemiol 1985:14:378-88.

31 Haupt CM, Alte D, Dörr $\mathrm{M}$, et al. The relation of exposure to shift work with atherosclerosis and myocardial infarction in a general population. Atherosclerosis 2008:201:205-11.

32 Laugsand LE, Vatten LJ, Platou $C$, et al. Insomnia and the risk of acute myocardial infarction: a population study. Circulation 2011;124:2073-81.

33 Boggild $\mathrm{H}$, Suadicani $\mathrm{P}$, Hein HO, et al. Shift work, social class, and ischaemic heart disease in middle aged and elderly men; a 22 year follow up in the Copenhagen Male Study. Occup Env Med 1999;56:640-5.

34 Rokkanen M, Uusitalo R. Changes in Job Stability: Evidence from Lifetime Job Histories. 2010. http://ftp.iza.org/dp4721.pdf

35 Harrington JM. Health effects of shift work and extended hours of work. Occup Environ Med 2001;58:68-72.

36 Albertsen K, Rafnsdóttir GL, Grimsmo A, et al. Workhours and worklife balance. Scand I Work Environ Health 2008;Suppl (5):14-21.

37 Jansen NW, Kant I, Nijhuis FJ, et al. Impact of worktime arrangements on work-home interference among Dutch employees. Scand J Work Environ Health 2004;30:139-48

38 Wennberg P, Lindahl B, Hallmans G, et al. The effects of commuting activity and occupational and leisure time physical activity on risk of myocardial infarction. Eur $J$ Cardiovasc Prev Rehabil 2006;13:924-30.

39 Hansson E, Mattisson K, Björk J, et al. Relationship between commuting and health outcomes in a cross-sectional population survey in southern Sweden. BMC Public Health 2011;11:834

40 Kiss P, De Meester M, Braeckman L. Differences between younger and older workers in the need for recovery after work. Int Arch Occup Environ Health 2008:81:311-20.

41 Gommans F, Jansen N, Stynen D, et al. The ageing shift worker: a prospective cohort study on need for recovery, disability, and retirement intentions. Scand J Work Environ Health 2015;41:356-67.

42 Kivimäki M, Jokela M, Nyberg ST, et al. Long working hours and risk of coronary heart disease and stroke: a systematic review and meta-analysis of published and unpublished data for 603838 individuals. Lancet 2015;386:1739-46.

43 Knauth P. Designing better shift systems. Appl Erg 1996;27:39-44. 


\section{Shift work and 20-year incidence of acute myocardial infarction: results from the Kuopio Ischemic Heart Disease Risk Factor Study}

Aolin Wang, Onyebuchi A Arah, Jussi Kauhanen and Niklas Krause

Occup Environ Med published online March 31, 2016

Updated information and services can be found at:

http://oem.bmj.com/content/early/2016/03/31/oemed-2015-103245

These include:

References This article cites 37 articles, 11 of which you can access for free at: http://oem.bmj.com/content/early/2016/03/31/oemed-2015-103245 \#BIBL

Email alerting

Receive free email alerts when new articles cite this article. Sign up in the service box at the top right corner of the online article.

\section{Notes}

To request permissions go to:

http://group.bmj.com/group/rights-licensing/permissions

To order reprints go to:

http://journals.bmj.com/cgi/reprintform

To subscribe to BMJ go to:

http://group.bmj.com/subscribe/ 